\title{
Communication
}

\section{Nanoliposomes Reduce Stroke Injury Following Middle Cerebral Artery Occlusion in Mice}

\author{
Saif Ahmad 1,2,", Seth Truran², Nina Karamanova², Adam Kindelin', Maria Lozoya ${ }^{3}$, Volkmar Weissig3, Daniel R. \\ Griffiths $^{2,4}$, Tyler Vail ${ }^{4}$, Jonathan Lifshitz ${ }^{2,4}$, Andrew F. Ducruet ${ }^{1,+}$ and Raymond Q. Migrino ${ }^{2,4,{ }^{,},+}$
}

1 Barrow Neurological Institute; saif.ahmad@commonspirit.org

2 Phoenix Veterans Affairs Health Care System; raymond.migrino@va.gov

3 Midwestern University; vweiss@midwestern.edu

4 University of Arizona College of Medicine-Phoenix; jlifshitz@arizona.edu

* Corresponding authors: A.S.: saif.ahmad@commonspirit.org; R.Q.M.:raymond.migrino@va.gov

+ A.F.D., R.Q.M. equal senior authors

\begin{abstract}
Neuroprotective strategies for stroke remain inadequate. Nanoliposomes comprised of phosphatidylcholine, cholesterol and monosialogangliosides (NL) induced an antioxidant protective response in endothelial cells exposed to amyloid insults. We tested the hypotheses that NL will preserve SH-SY5Y neuroblastoma cell viability following hypoxic injury and will reduce injury in mice following middle cerebral artery occlusion (MCAO). Neuroblastoma were exposed to 20-hour physoxic ( $5 \%$ oxygen) or hypoxic ( $1 \%$ oxygen) condition without or with NL (100 or $300 \mu \mathrm{g} / \mathrm{mL}$ ). Viability was measured using calcein-AM fluorescence and SH-SY5Y gene expression of antioxidant proteins heme oxygenase-1 (HO-1), $\mathrm{NAD}(\mathrm{P}) \mathrm{H}$ quinone dehydrogenase 1 (NQO1) and superoxide dismutase 1 (SOD1) were measured by quantitative polymerase chain reaction. C57BL/6J mice were treated with saline $(\mathrm{N}=8)$ or $\mathrm{NL}(10000 \mathrm{ug} / \mathrm{mL}, \mathrm{N}=7)$ while undergoing 60-minute MCAO followed by reperfusion. Day 2 post-injury neurologic impairment score and infarction size were compared. Neuroblastoma showed reduced viability following hypoxia that was reversed by NL. NL increased gene expression of HO-1, NQO1 and SOD1 versus controls. NL-treated mice showed reduced neurologic impairment and brain infarct size $(18.8 \pm 2 \%$ versus $27.3 \pm 2.3 \%, \mathrm{p}=0.017)$ versus controls. NL reduced stroke injury in mice subjected to MCAO likely through induction of an antioxidant stress response. NL is a candidate novel agent for stroke.
\end{abstract}

Keywords: keyword 1; stroke 2; liposome 3; cerebrovascular disease 4; oxidative stress 5; dementia

\section{Introduction}

Stroke is the second leading cause of death and the third leading cause of disabilityadjusted, life years worldwide ${ }^{1}$. Stroke is also a major factor in later development of vascular dementia and Alzheimer's disease ${ }^{1}$. Despite the widespread application of two reperfusion strategies (intravenous thrombolytics and endovascular thrombectomy), post-ischemic brain injury still results in significant long-term disability and increased health care costs in the large population of stroke survivors ${ }^{2}$. Prolonged ischemia may also reduce or abolish the benefits of reperfusion therapy and delayed reperfusion engenders secondary reperfusion injury ${ }^{3}$. Therefore the National Institute of Neurologic Disorders and Stroke (NINDS) Stroke Progress Review group has called for the development of adjuvant therapies to protect against reperfusion injury as well as to extend the therapeutic window of reperfusion therapy ${ }^{4}$. Nanoliposomes comprise $<100 \mathrm{~nm}$ sized collection of phospholipids, and cargo-free nanoliposome formulation comprised of cholesterol, phosphatidylcholine and monosialoganglioside $(\mathrm{NL})$ were shown to protect against oxidative and nitrative stress induced in human vascular tissue and endothelial cells by amyloidogenic light chain ${ }^{5}$ and medin proteins ${ }^{6}$. NL fully reversed medin-induced endothelial cell injury through nuclear factor erythroid 2-related factor 2 (Nrf2)-mediated activation of cellular antioxidant protective responses, with increased production of antioxidants heme oxygenase-1 (HO-1), NAD(P)H quinone dehydrogenase 1 (NQO1) and 
superoxide dismutase-1 (SOD1). In light of these antioxidant effects, we aim to test the efficacy of NL in mitigating hypoxic injury both in vitro and in vivo. We tested the hypothesis that NL will preserve viability of human neuroblastoma and brain microvascular endothelial cells exposed to hypoxia and that NL will reduce functional and histologic brain damage in mice subjected to middle cerebral artery occlusion stroke.

\section{Results}

\subsection{Nanoliposomes}

Nanoliposomes comprised of phosphatidylcholine, cholesterol and monosialoganglioside $(70 \%, 25 \%, 5 \%$ molar ratios, respectively) were measured to be $38.83 \pm 1.23 \mathrm{~nm}$ in size with polydispersity index of 0.32 and Z-potential of $-8.76 \mathrm{mV}$.

\subsection{Neuroblastoma}

Neuroblastoma cells exposed to hypoxia showed significantly reduced viability compared to physoxic control, while co-treatment with low $(100 \mu \mathrm{g} / \mathrm{ml})$ or high $(300 \mu \mathrm{g} / \mathrm{ml})$ dose of NL improved cell viability with full restoration at high dose (Figure 1A). There was no significant difference in gene expression of HO-1, NQO1 or SOD1 between neuroblastoma cells exposed to hypoxia or physoxia, but co-treatment with NL $(300 \mu \mathrm{g} / \mathrm{ml}) \mathrm{sig}$ nificantly increased HO-1, NQO1 and SOD 1 gene expression in cells exposed to hypoxia (Figure 1B-D). Human brain microvascular endothelial cells showed reduced viability after exposure to hypoxia with restoration of viability when treated with NL at 100or 300 $\mu \mathrm{g} / \mathrm{ml}$ (Figure 1E).
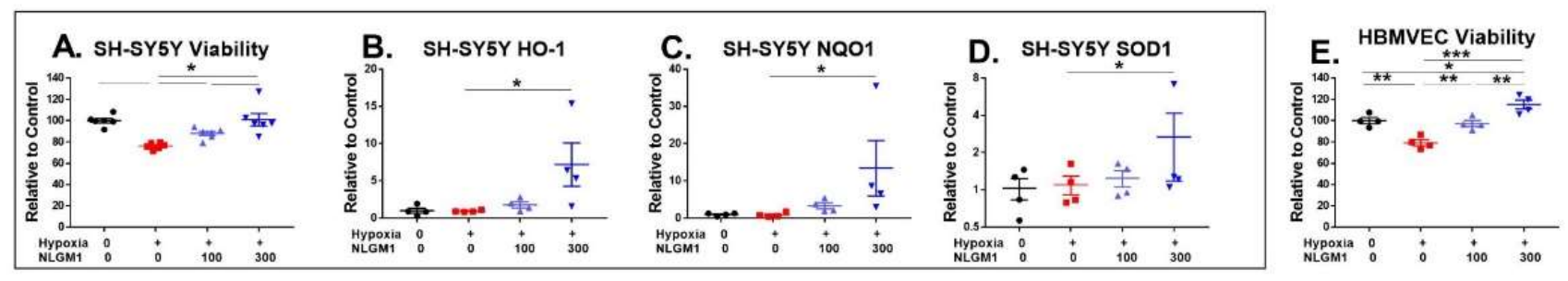

Figure 1. Hypoxic injury to human neuroblastoma and brain microvascular endothelial cells. A. SH-SY5Y cells exposed to hypoxia for 20 hours showed reduced viability that was restored by treatment with NL $100 \mu \mathrm{g} / \mathrm{ml}$ and $300 \mu \mathrm{g} / \mathrm{ml}$, respectively. Hypoxia did not elicit any change in gene expression measured by quantitative polymerase chain reaction of HO-1 (B), NQO1 (C) and SOD1 (D) in SH-SY5Y cells, but treatment with NL $300 \mu \mathrm{g} / \mathrm{mL}$ showed significant increase in gene expression of the antioxidant enzymes. E shows similar response in human brain microvascular endothelial cells (HBMVEC) with reduced viability with hypoxia and restoration of viability with NL treatment. ${ }^{*} \mathrm{p}<0.05,{ }^{* *} \mathrm{p}<0.01$, ${ }^{* * *} \mathrm{p}<0.001$. 


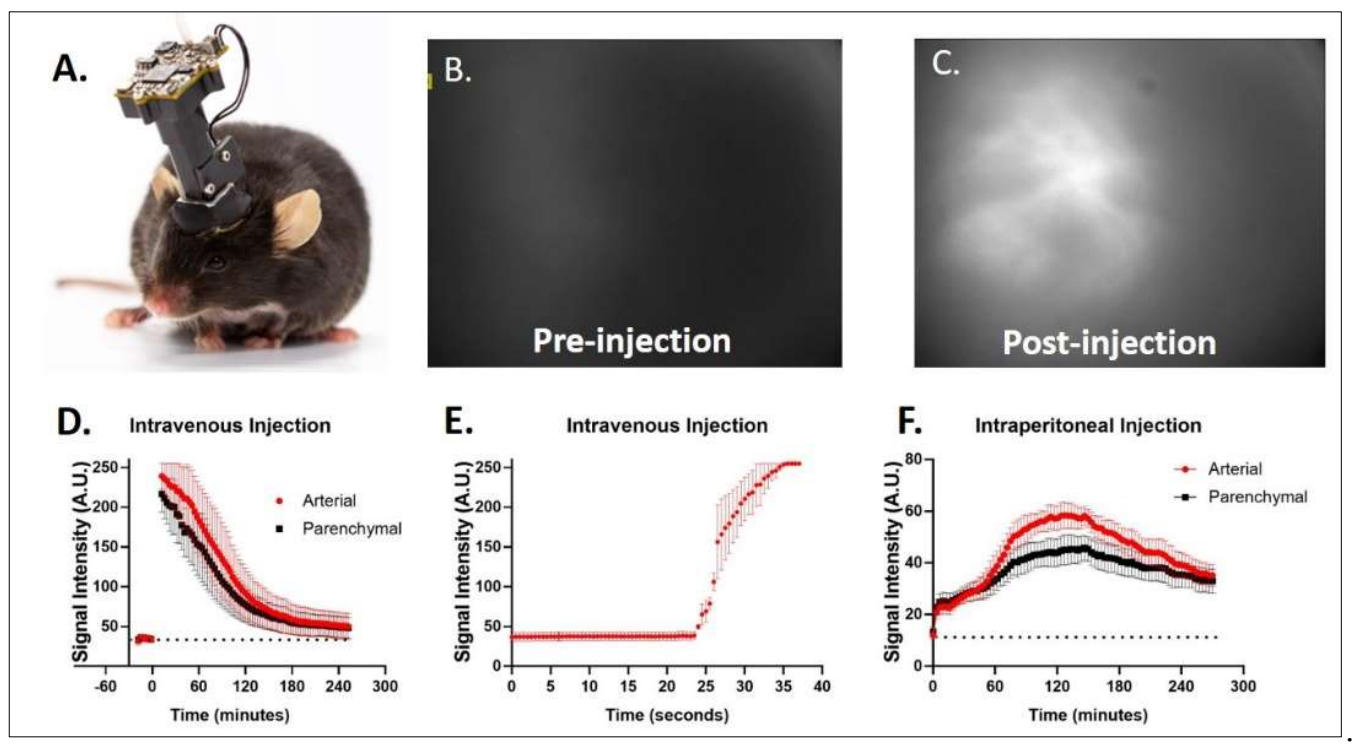

Figure 2. In vivo administration of NL. A. Mouse with cranial window and Miniscope camera. B-C. Fluorophore-labelled NL were injected intravenously in a mouse and fluorescent signal was measured; B shows brain prior to injection and $\mathrm{C}$ shows brain at peak fluorescent signal. D-F shows the time course of fluorescent signal in cerebral artery (red) and parenchymal brain regions (black) following intravenous (D-E) and intraperitoneal (F) administration, (N=3). Note that signal saturates (maximum reading) at 256 arbitrary units (A.U.). E shows NL signal during initial period following tail vein injection demonstrating NL delivery to cerebrovasculature within 30 seconds of injection.

\subsection{In vivo NL administration}

NL administration showed delivery to mouse cerebral arteries with persistence of signal for up to 2 hours for IV injection and for more than 4 hours for IP injection (Figure 2, Movie Supplement 1 and 2). Following tail vein injection, NL was noted to reach cerebral circulation within 30 seconds of injection (Figure 2E).

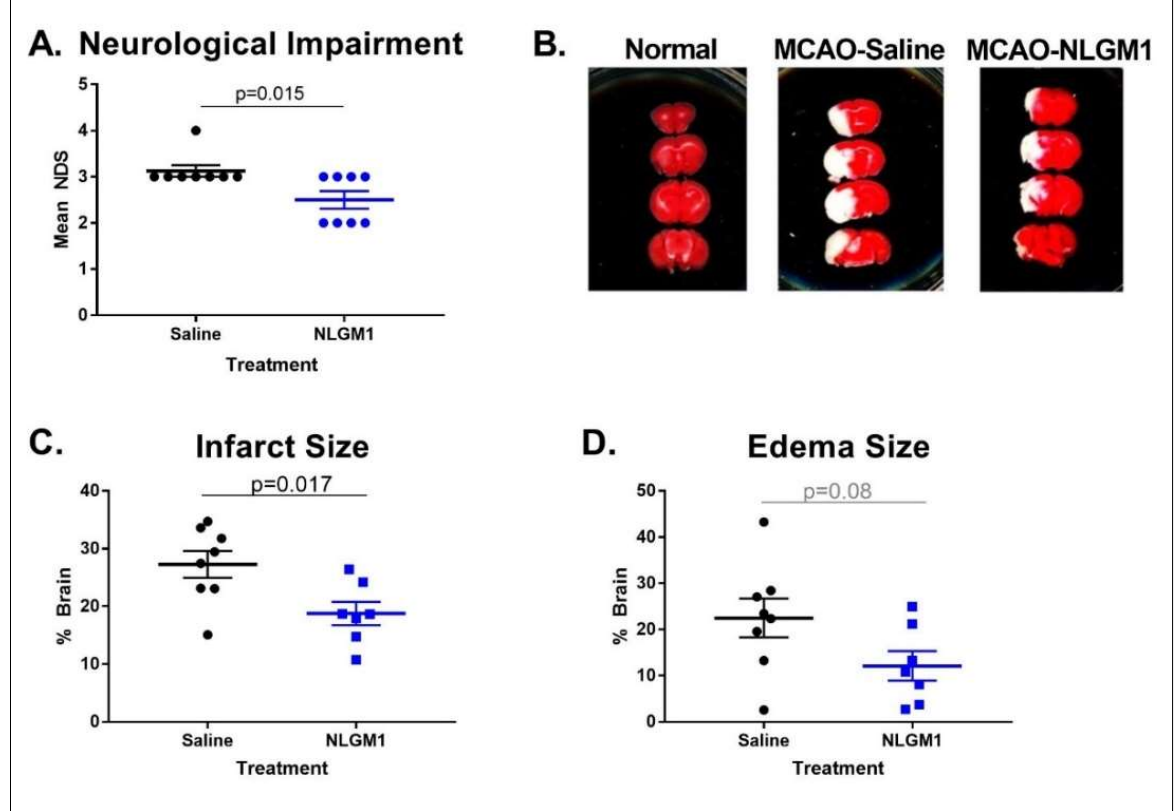

Figure 3. NL reduced stroke injury following MCAO. Mice treated with NL during MCAO showed less neurological impairment compared to saline-treated controls (A). B shows representative 
brain sections after triphenyltetrazolium chloride staining. C-D show that mice who had MCAO treated with NL had smaller infarcts with a trend towards reduced brain edema in NL-treated mice.

\subsection{Stroke Injury}

Mice that received NL showed improved neurologic impairment score compared to saline control (Figure 3A). They also had significantly smaller infarcts and a trend towards decreased brain edema (Figure 3B-D).

\section{Discussion}

This is an initial proof-of-concept demonstration that nanoliposomes comprised of phosphatidylcholine, cholesterol and monosialoganglioside could mitigate functional and structural stroke injury in an established preclinical ischemia model.

Stroke represents the second most frequent cause of death in people older than 60 years old, the most frequent cause of permanent disability and the second leading cause of dementia ${ }^{7}$. Ischemic stroke from cerebral ischemia due to cerebral artery occlusion accounts for $85 \%$ of all strokes ${ }^{8}$. Immediately after ischemia, neurons are unable to sustain their normal transmembrane ionic gradient and homeostasis leading to oxidative and nitrative stress, excitotoxicity, cell death and neuronal destruction' 9 . While the predominant emphasis is to reverse hypoperfusion, minimize tissue infarct and salvage the ischemic penumbra through reperfusion therapy, there is still great need for neuroprotective approaches that could enhance these revascularization strategies or administered in case revascularization is no longer an option. Oxidative stress in stroke results from enzymatic generation of free radicals fueled by oxygen supply which still reaches the ischemic tissue in the penumbra particularly after reperfusion ${ }^{7}$. Neutralizing oxidative stress is a promising strategy as the ischemic brain is susceptible to oxidative damage due to high oxygen consumption and relatively low antioxidant capacity ${ }^{7}$. Antioxidant agents such as edavarone that scavenges hydroxyl, peroxyl and superoxide radicals have been shown in cell and preclinical models to be efficacious, although this has not yet convincingly benefited patients with acute ischemic stroke ${ }^{10}$. In human vascular models of amyloid toxicity mediated by oxidative and nitrative stress, NL was shown to be effective in preventing endothelial dysfunction and endothelial cell death following exposure to amyloidogenic light chain and aging-associated medin proteins ${ }^{5,6}$. Our results show that the protective effect conferred by NL extends to preserving viability of human brain microvascular endothelial cells and neuroblastoma cells exposed to hypoxic insult. In our prior work, we showed that NL's ability to preserve cellular viability in the setting of amyloid-induced oxidative stress was due to increased gene and protein expression of endogenous antioxidants HO-1, NQO1 and SOD1 through Nrf2-mediated signaling ${ }^{6}$. HO- 1 is an important enzymatic defense against oxidative stress through cleavage of heme to yield physiological antioxidants biliverdin and bilirubin ${ }^{5,11}$. NQO1is an antioxidant enzyme through promotion of 2-electron reductions of quinones and depression of quinone levels leading to reduced generation of reactive oxygen intermediates by redox cycling 5 , 12. SOD1 catalyzes the dismutation of superoxide anion ${ }^{6}$. Our results show that neuroblastoma cells exposed to hypoxic condition do not generate an increase in gene expression of these antioxidant enzymes, but treatment with NL does, accompanied by preservation of cell viability to levels similar to physoxia condition. In vivo, administration of NL similarly resulted in reduced functional impairment and brain infarct size in mice exposed to MCAO followed by reperfusion. The protective effect of $\mathrm{NL}$ in the mouse stroke model is likely related, at least in part, to its ability to protect neuronal and endothelial cells in the face of hypoxic insult as demonstrated in our in vitro cell model, although this has yet to be empirically established in vivo.

Miniscope imaging provided pharmacokinetic insight that the NL formulation used persists in cerebrovascular circulation 2 hours or longer following either IV or IP 
administration, suggesting sufficient exposure time to brain tissue within the therapeutic window required to treat stroke patients.

The data are consistent with prior reports of mitigation of stroke injury by monosialoganglioside in a rodent model published by Li and colleagues ${ }^{13}$, although monosialoganglioside treatment had equivocal results in human stroke clinical trials ${ }^{14,15}$. Important differences with our study are that we injected nanoliposomes and our formulation was composed of 3 phospholipids: phosphatidylcholine, cholesterol and monosialoganglioside with the latter comprising only $5 \%$ molar weight. The prior study by Li and colleagues using monosialoganglioside resulted in $6.8 \%$ absolute reduction of infarction volume that was achieved using $150 \mathrm{mg} / \mathrm{kg}$ total dose ${ }^{13}$, whereas our NL formulation with $\sim 8.4 \mathrm{mg} / \mathrm{kg}$ monosialoganglioside component resulted in $8.5 \%$ absolute reduction of infarction volume. $\mathrm{Li}$ and his team injected free monosialoganglioside in saline; due to its low solubility in aqueous medium this glycolipid forms micellar aggregates the sizes of which were not reported. It is likely that any uncontrolled aggregate formation can severely limit the bioavailability of monosialogangliosides that could in turn reduce its efficacy. This might explain the fact that we needed much less of the monosialogangliosides in our nanoliposomes to produce the protective effect. Nevertheless, our NL formulation needs to be tested for efficacy in future translational studies.

The study has several limitations. Only male mice were tested and follow up tests should include female mice. The ideal dosing and timing of NL administration was not determined, but promising results in this initial study serve as a solid foundation to base future optimization experiments. The first dose was given 1 hour prior to arterial occlusion (hence allowing the agent to potentially affect brain cells prior to ischemia) and a second dose given following occlusion and just before reperfusion. This timing is not applicable to the most common clinical scenario wherein the treatment window available is only following occlusion (onset of stroke). Therefore, NL protective effect would have to be tested in the future within this window. It is important to note however that strokes are frequently accompanied by prodromal transient ischemic attacks representing symptomatic but brief flow disruptions not leading to irreversible injury. In such cases, the NL treatment scheduling we employed in this model would apply, so our chosen mode/timing of administration does have some valid translational clinical correlate.

\section{Materials and Methods}

\section{Nanoliposome production}

The details of the production of NL have previously been described ${ }^{5,6}$. NL used for treatment was prepared from phosphatidylcholine, cholesterol and monosialoganglioside (70:25:5 \% molar ratios) using lipid film hydration method. The hydrodynamic diameters of the liposomal nanoparticles were measured by dynamic light scattering (DLS) in triplicates using a Malvern Nano ZS Zetasizer (Malvern Panalytical Inc, Westborough, MA, USA) at $25^{\circ} \mathrm{C}$. In this study, the size reported is based on average particle diameter by volume. The zeta potential values of the NLGM1 suspensions were measured in disposable cuvettes equipped with gold electrodes. Zeta potential was determined using electrophoretic light scattering and is reported as the Z-average. Each measurement was performed on freshly prepared samples without dilutions.

\section{Hypoxic injury}

SH-SY5Y human neuroblastoma cells (ECACC General Collection, Public Health England, passages 16-18) were seeded into 6-well plates and each group was exposed to the following conditions for 20 hours using Bio Tek Cytation 5 (Fisher Scientific, Waltham MA): physoxia ( $5 \%$ oxygen, $5 \%$ carbon dioxide, $90 \%$ nitrogen), hypoxia ( $1 \%$ oxygen, $5 \%$ carbon dioxide, $94 \%$ nitrogen), hypoxia co-treated with NL $(100 \mu \mathrm{g} / \mathrm{mL})$ or 
hypoxia co-treated with NL $(300 \mu \mathrm{g} / \mathrm{mL}$, the dose chosen as it was the dose found to protect endothelial cells against medin and light chain amyloid-induced oxidative stress ${ }^{5,6}{ }^{6}$. Cells were then given calcein-acetoxymethyl (10 nmol/L, Life Technologies), a fluorescent probe that measures cell viability through detection of active and intact esterases 16 and cell viability was measured using flow cytometer (Beckman Coulter FC500, Indianapolis IN) at 494/517 $\mathrm{nm}$ excitation/emission settings ${ }^{6}$. Separate groups were exposed to same treatment conditions and gene expression was measured following lysis, ribonucleic acid extraction and conversion to complementary deoxyribonucleic acid using Aurum Total RNA Mini Kit and iScript cDNA synthesis kit (Bio-Rad Laboratories, Hercules CA) as per previous methods ${ }^{6}$. Primers for HO-1 (SEQ1 F-5'-GAAGACACCCUAAUGUGGCAGCTG-3' R-3'-GACCUUCUGUGGGAUUACACCGUCGAC-5' SEQ2 F-5'-ACAACAUUGUCUGAUAGUAGCUUGA-3' R-3'-UUUGUUGUAACAGACUAUCAUCGAACU-5') and SOD1 (SEQ1 F-5'-CCTCGGAACCAGGACCT-3' SEQ2 F-5'TTAATGCTTCCCCACACCTT-3') were obtained from IDT DNA Technologies (Coralville IA) and NQO1 (F - 5'-ATGTATGACAAAGGACCCTTCC-3' R-3'-GGTACATGAGAGACGTTCCCT-5') was obtained from Sigma-Aldrich. $\beta$-actin served as reference normalization gene. In separate experiments, human brain microvascular endothelial cells (CellBiologics, Chicago IL, passages 5-8) were exposed for 20 hours to hypoxia (1\% oxygen) without or with NL (100 or $300 \mu \mathrm{g} / \mathrm{ml})$ and viability was compared with control endothelial cells in incubator (room air) using calcein-AM flow cytometry.

In vivo testing of NL administration

The animal experiments were approved and were under the supervision of the Institutional Animal Care and Use Committee of the University of Arizona College of Medicine-Phoenix and Barrow Neurological Institute at St. Joseph's Hospital and Medical Center and conform to the Animal Welfare Act and applicable statutes. First, we tested delivery and persistence of NL in mouse cerebral circulation by producing a fluorophore-containing modification of NL (phosphatidylcholine, monosialoganglioside, 1,2dioleoyl-sn-glycero-3-phosphoethanolamine-N-carboxyfluorescein in 85:5:10 \% molar ratios) visualized with a miniature fluorescent microscope (V3 Miniscope) secured to the skull. Three C57BL/6J male mice (8 weeks old) underwent surgery to attach a Miniscope, with details as previously described ${ }^{17}$. A single GRIN lens (Edmund Optics, \#64-538) was implanted perpendicular to the parieto-temporal cortex. Seven days after surgery, each mouse was anesthetized and secured in a stereotaxic frame for imaging. Baseline images were acquired and then the mouse received $0.1 \mathrm{ml}$ of $\mathrm{NL}(10000 \mu \mathrm{g} / \mathrm{ml})$ via tail vein intravenous (IV) injection. All imaging was done with modified Miniscope software with identical capture settings (Achromatic lens: 7.5mm, FPS: 30, Exposure: 100\%, Gain: 0, LED: $100 \%)$ capturing continuous video $(30 \mathrm{~Hz})$ modified to capture time lapse video (1 image per 1-5 minutes) turning the LED to $0 \%$ between image acquisitions. After 1day recovery, the process was repeated following injection of $0.1 \mathrm{ml}$ of NL via intraperitoneal (IP) injection. Fluorescent signals from 2 regions of interest (cerebral artery and brain parenchyma) were measured using ImageJ (National Institutes of Health, Bethesda MD) and plotted by time.

\section{Middle cerebral artery occlusion injury}

20 week old male C57BL/6J mice (Jackson Laboratory, Bar Harbor ME) underwent transient MCAO as previously described ${ }^{18}$. In brief, a silicon-coated 6-0 nylon suture was introduced into the external carotid artery and advanced up to the internal carotid artery to occlude the middle cerebral artery for 60 minutes followed by removal of the filament to restore perfusion. The mice were divided into two groups: an experimental group received NL (phosphatidylcholine, cholesterol, monosialoganglioside in 70:25:5 \% molar ratios, $10000 \mu \mathrm{g} / \mathrm{ml}, \mathrm{N}=7$ ) intraperitoneally (IP) 1 hour prior to occlusion and 
intravenously (IV) just before reperfusion, while the control group $(\mathrm{N}=8)$ received saline IP and IV at the same time points.

Neurological score, infarction volume and edema analyses

Neurological deficit scoring (NDS) was performed according to the modified Bederson scale 48 hours after stroke, similar to previous methods ${ }^{19}, 20$. In brief, the neurological score was measured using a 5-point scale (0-No neurologic deficit; 1-Failure to extend left forepaw fully; 2- Circling to the left indicates a moderate focal neurologic deficit; 3- Falling to the left shows severe focal deficit; 4- Inability to walk spontaneously and diminished level of consciousness). Infarction volume was assessed 48 hours post MCAO. In brief, mice were anesthetized and brains were harvested for coronal sections ( $2 \mathrm{~mm}$ thickness) by using a mouse brain matrix (Roboz Surgical Instrument, Gaithersburg MD). Brain slices were then stained with 1\% solution of 2,3,4-triphenyl tetrazolium chloride (Sigma-Aldrich) at $37 \pm 0.5^{\circ} \mathrm{C}$ for $15-20$ minutes. Using ImageJ, corrected infarct volume was calculated as previously reported ${ }^{18}$. An algorithm described by McBride, et al. ${ }^{21}$ was applied to indirectly calculate infarct volumes, corrected for edema, as well as the degree of brain edema. NDS and infarction measurements were performed by an investigator blinded to treatment allocation (AK).

\section{Data and Statistical Analyses}

Cell viability and gene expression values were compared using one-way repeated measures analysis of variance (ANOVA) with post-hoc pairwise comparison using Holm-Sidak method for normally distributed data with equal variance, or repeated measures ANOVA on ranks with post-hoc pairwise comparison using Tukey test for data that are not normally distributed or with unequal variance (Sigma Stat 3.5, Systat, San Jose CA) . Neurologic function and histopathology were compared using unpaired Student's t-test between drug-treated and control groups. Significant p-value was set at 0.05 (two-sided). Data are presented as mean \pm standard error of means.

\section{Conclusions}

Nanoliposomes composed of phosphatidylcholine, cholesterol and monosialogangliosides prevented hypoxia-induced injury in neuroblastoma and endothelial cells and reduced functional and structural brain damage in mice exposed to middle cerebral artery occlusion. NL is a promising candidate for further testing as a neuroprotective adjuvant agent in stroke.

\section{Patent}

A patent application has been submitted by the Department of Veterans Affairs resulting from the work reported in this manuscript.

Supplementary Materials: Video S1: Intravenous injection of nanoliposomes, Video S2: Intraperitoneal injection of nanoliposomes.

Author Contributions: Conceptualization, R.Q.M, A.F.W., V.W. and J.L.; methodology, S.A., S.T., N.K., A.K., M.L., D.R.G., T.V.; formal analysis, S.A., A.F.W., R.Q.M., D.R.G., T.V.; writing-original draft preparation, R.Q.M., S.A., S.T., M.L., D.R.G.; writing-review or editing, all authors; supervision, A.F.D., R.Q.M., V.W., J.L.; project administration, R.Q.M., A.F.D.; funding acquisition, R.Q.M. All authors have read and agreed to the published version of the manuscript.

Funding: This research was funded by the Veterans Affairs BLRD Merit BX003767 and Department of Defense W81XWH-17-1-0473. The content and views of this manuscript do not represent the views of the United States Department of Veterans Affairs or the United States government.

Institutional Review Board Statement: The study was conducted according to the guidelines of the Declaration of Helsinki and approved by the Institutional Review Boards of St. Joseph's Hospital/Barrow Neurological Institute (protocol number 540 approved 2/22/2020 and 2/3/2021) and University of Arizona College of Medicine-Phoenix (protocol number 13-460 approved 8/28/2019). 
Data Availability Statement: Data are available upon written request to corresponding author in compliance with applicable policies of the Department of Veterans Affairs.

Acknowledgments: We would like to thank Ms. Gail Farrell and the Phoenix VA Office of Research for administrative support.

Conflicts of Interest: The authors declare no conflict of interest. The funders had no role in the design of the study; in the collection, analyses, or interpretation of data; in the writing of the manuscript, or in the decision to publish the results.

\section{References}

1. Vijayan M, Reddy PH. Stroke, Vascular Dementia, and Alzheimer's Disease: Molecular Links. J Alzheimers Dis 2016; 54(2): 427-43.

2. Benjamin EJ, Virani SS, Callaway CW, Chamberlain AM, Chang AR, Cheng S et al. Heart Disease and Stroke Statistics-2018 Update: A Report From the American Heart Association. Circulation 2018; 137(12): e67-e492.

3. Nour M, Scalzo F, Liebeskind DS. Ischemia-reperfusion injury in stroke. Interv Neurol 2013; 1(3-4): 185-99.

4. Saver JL, Albers GW, Dunn B, Johnston KC, Fisher M, Consortium SV. Stroke Therapy Academic Industry Roundtable (STAIR) recommendations for extended window acute stroke therapy trials. Stroke 2009; 40(7): 2594-600.

5. Franco DA, Truran S, Weissig V, Guzman-Villanueva D, Karamanova N, Senapati S et al. Monosialoganglioside-Containing Nanoliposomes Restore Endothelial Function Impaired by AL Amyloidosis Light Chain Proteins. J Am Heart Assoc 2016; 5(6).

6. Karamanova N, Truran S, Serrano GE, Beach TG, Madine J, Weissig V et al. Endothelial Immune Activation by Medin: Potential Role in Cerebrovascular Disease and Reversal by Monosialoganglioside-Containing Nanoliposomes. J Am Heart Assoc 2020; 9(2): e014810.

7. Chamorro A, Dirnagl U, Urra X, Planas AM. Neuroprotection in acute stroke: targeting excitotoxicity, oxidative and nitrosative stress, and inflammation. Lancet Neurol 2016; 15(8): 869-881.

8. Khoshnam SE, Winlow W, Farzaneh M, Farbood Y, Moghaddam HF. Pathogenic mechanisms following ischemic stroke. Neurol Sci 2017; 38(7): 1167-1186.

9. Lo EH, Moskowitz MA, Jacobs TP. Exciting, radical, suicidal: how brain cells die after stroke. Stroke 2005 ; 36(2): 189-92.

10. Lapchak PA. A critical assessment of edaravone acute ischemic stroke efficacy trials: is edaravone an effective neuroprotective therapy? Expert Opin Pharmacother 2010; 11(10): 1753-63.

11. Kikuchi G, Yoshida T, Noguchi M. Heme oxygenase and heme degradation. Biochem Biophys Res Commun 2005; 338(1): 55867.

12. Dinkova-Kostova AT, Talalay P. NAD(P)H:quinone acceptor oxidoreductase 1 (NQO1), a multifunctional antioxidant enzyme and exceptionally versatile cytoprotector. Arch Biochem Biophys 2010; 501(1): 116-23.

13. Li L, Tian J, Long MK, Chen Y, Lu J, Zhou C et al. Protection against Experimental Stroke by Ganglioside GM1 Is Associated with the Inhibition of Autophagy. PLoS One 2016; 11(1): e0144219.

14. Candelise L, Ciccone A. Gangliosides for acute ischaemic stroke. Cochrane Database Syst Rev 2001; (4): CD000094.

15. Zhang W, Krafft PR, Wang T, Zhang JH, Li L, Tang J. Pathophysiology of Ganglioside GM1 in Ischemic Stroke: Ganglioside GM1: A Critical Review. Cell Transplant 2019; 28(6): 657-661.

16. Neri S, Mariani E, Meneghetti A, Cattini L, Facchini A. Calcein-acetyoxymethyl cytotoxicity assay: standardization of a method allowing additional analyses on recovered effector cells and supernatants. Clin Diagn Lab Immunol 2001; 8(6): 11315.

17. Ghosh KK, Burns LD, Cocker ED, Nimmerjahn A, Ziv Y, Gamal AE et al. Miniaturized integration of a fluorescence microscope. Nat Methods 2011; 8(10): 871-8.

18. Zhao XJ, Larkin TM, Lauver MA, Ahmad S, Ducruet AF. Tissue plasminogen activator mediates deleterious complement cascade activation in stroke. PLoS One 2017; 12(7): e0180822.

19. Rynkowski MA, Kim GH, Garrett MC, Zacharia BE, Otten ML, Sosunov SA et al. C3a receptor antagonist attenuates brain injury after intracerebral hemorrhage. J Cereb Blood Flow Metab 2009; 29(1): 98-107.

20. Hoda MN, Bhatia K, Hafez SS, Johnson MH, Siddiqui S, Ergul A et al. Remote ischemic perconditioning is effective after embolic stroke in ovariectomized female mice. Transl Stroke Res 2014; 5(4): 484-90.

21. McBride DW, Klebe D, Tang J, Zhang JH. Correcting for Brain Swelling's Effects on Infarct Volume Calculation After Middle Cerebral Artery Occlusion in Rats. Transl Stroke Res 2015; 6(4): 323-38. 